\title{
Parameter estimation of support vector machine with radial basis function kernel using grid search with leave-p-out cross validation for classification of motion patterns of subviral particles
}

\begin{abstract}
The classification of subviral particle motion in fluorescence microscopy video sequences is relevant to drug development. This work introduces a method for estimating parameters for support vector machines (SVMs) with radial basis function (RBF) kernels using grid search with leave-pout cross-validation for classification of subviral particle motion patterns. RBF-SVM was trained and tested with a large number of combinations of expert-evaluated training and test data sets for different RBF-SVM parameters using grid search. For each subtest, the mean and standard deviation of the accuracy of the RBF-SVM were calculated. The RBF-SVM parameters are selected according to the optimal accuracy. For the optimal parameters, the accuracy is $89 \% \pm 13 \%$ for $N=$ 100. Using the introduced computer intensive machine learning parameter adjustment method, an RBF-SVM has been successfully trained to classify the motion patterns of subviral particles into chaotic, moderate and linear movements.
\end{abstract}

Keywords: Grid search, leave-p-out cross-validation, parameter estimation, principal component analysis, subviral particles, support vector machine

https://doi.org/10.1515/cdbme-2021-2031

\section{Introduction}

Despite many positive aspects, advancing globalization also holds risks. The current Covid-19 pandemic demonstrates the

\footnotetext{
* Corresponding author: Ricardo M. Schuhmann: Institute for Biomedical Engineering (IBMT), Faculty of Life Science Engineering (LSE), Technische Hochschule Mittelhessen (THM) University of Applied Sciences, Gießen, Germany, email: ricardo.mario.schuhmann@Ise.thm.de

Andreas Rausch, Thomas Schanze: Technische Hochschule Mittelhessen, Gießen, Germany
}

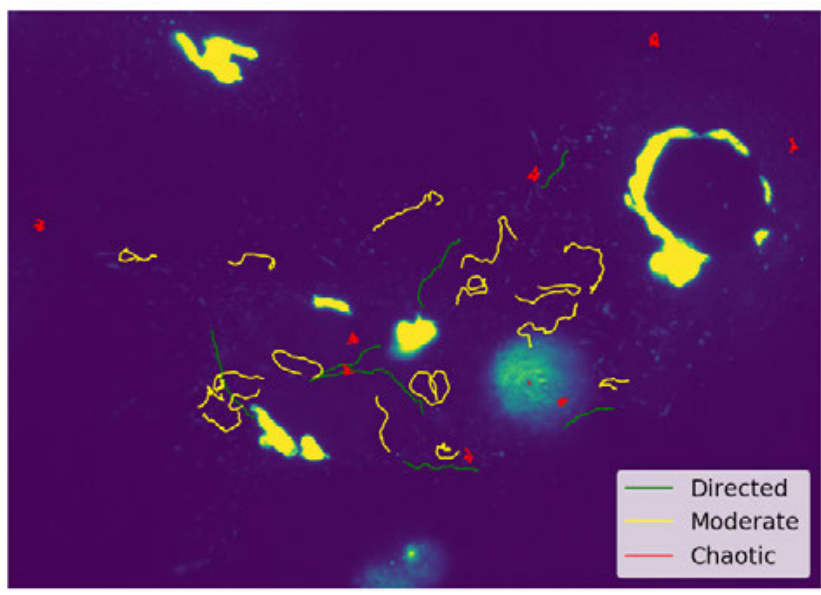

Figure 1: Image sequences from an innovative fluorescence livecell imaging method visualize intracellular processes [2]. Bright green spots are a result of high density of fluorescent medium. A virological expert manually selected and classified 33 subviral particle tracks on basis of these images sequences into directed, moderate and chaotic motion patterns.

risk of fast globally spreading viral diseases. All the more reason for virus research to come to the fore. Another thread to the human population are pathogens of hemorrhagic fevers, like the Ebola virus that has a mortality rate of about $50 \%$ [1]. To advance the development of new drugs, a profound knowledge of the pathogens' subviral particles is mandatory. Especially, the effect of experimental drugs onto the subviral particle motion behavior holds important information for pharmaceutical research. The cells analyzed for this work were infected with the Marburg virus, whose properties are similar compared to those of the Ebola virus. For this purpose, image sequences have been provided by scientists from the Institute of Virology at the Philipps-University, Marburg, which originate from an innovative fluorescence microscopic livecell imaging method to visualize intracellular processes [2]. Algorithms to automatically track subviral particles in fluorescence images were introduced by Kienzle et al. [3] and further developed by Rausch et al. [4]. 
Some basics of this work have already been presented by Schuhmann et al. [5]. The developed method allows the classification of movement patterns of subviral particles using a support vector machine (SVM). It was proven that the concept enables a discrimination between directed, moderate and chaotic motion patterns (Fig. 1). This work builds on these findings and introduces a method for estimating parameters for support vector machines with radial basis function (RBF) kernels using grid search with leave-p-out cross-validation for classification of subviral particle motion patterns.

\section{Methods}

In the previously published work by Schuhmann et al. [5], tracking coordinates and corresponding metadata are used to define parameters that quantify the movement patterns of subviral particles in space and time. The parameters fractal dimension [6], straightness (ratio of displacement and length), curvature [7] and the results of the contrast function from the mean square deviation [8] refer to the spatial extent of the track. In addition, the acceleration and speed describe the properties of the particle motion and consider not only the space but also the dimension of time. For this purpose, the averaged speed and the averaged acceleration were calculated over one track each. The speed results from the quotient of the distance traveled by a subviral particle and the time required to travel it. The acceleration is considered twice: As absolute and as non-absolute value to include negative acceleration. The particles' speed is additionally calculated using a sliding window of size 2 and 10 . Thus, the speed is calculated between each 2 nd respectively 10th coordinate while skipping the coordinates in between. This definition of speed is more robust against back and forth movements of subviral particles. Principal component analysis (PCA) is applied to represent and condense the information content of all parameters. Model building for classification is performed by SVM. It bases on a dataset whose 33 tracks have been selected and classified in advance by a virologist at the Institute of Virology, PhilippsUniversity of Marburg (Fig. 1). New tracks can be included in the principal components by the scalar product of eigenvectors and the parameter values of the new track. Their class can be predicted with the created SVM model.

In the previous stages of the work [5] a SVM with linear kernel was applied. This work focuses on the application of the RBFSVM [9]. To optimize the accuracy of the decision function of the RBF-SVM the parameters $C$ and $\gamma$ need to be chosen carefully. $C$ is the so-called penalty parameter, which controls the accepted errors. $\gamma$ determines how large the influence of

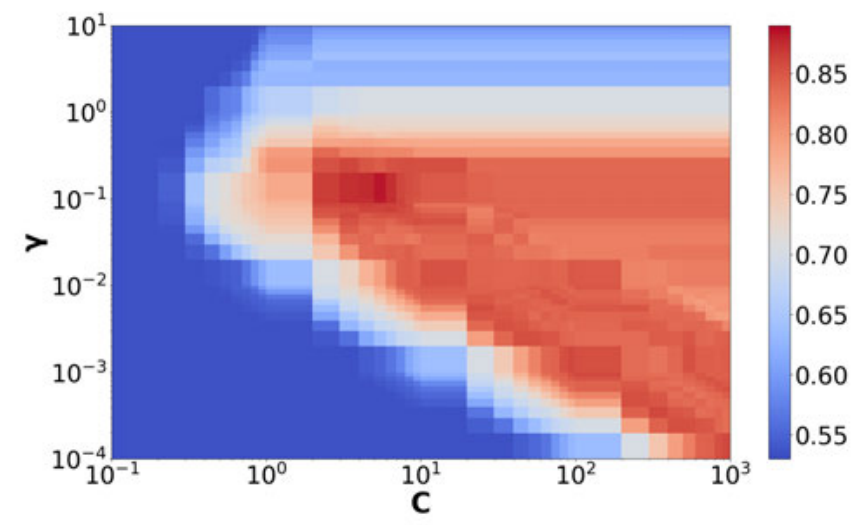

Figure 2: RBF-SVM parameter dependent average accuracies for $N=100$ are shown by means of a color heat map as a function of the RBF-SVM parameters for $0.1 \leq C \leq 1000$ and $0.0001 \leq \gamma \leq$ 10. Dark red areas represent combinations of $\gamma$ and $C$ that lead to a high accuracy.

each track is and thus affects the curvature of the decision function. For parameter estimation, a grid search is performed in combination with leave-p-out (LPO) cross-validation (CV) [10] on the RBF-SVM. For this purpose, the data set is split into training and test data for $p=7$ according to the oftenused ratio 80/20 with a number of $N=100$ random combinations. The grids are chosen with parameter space for $C \in\left\{x \cdot 10^{y} \mid x \in\{1,2, \ldots, 10\} \wedge y \in \mathbb{Z} \wedge-1 \leq y \leq 2\right\} \quad$ and $\gamma \in\left\{x \cdot 10^{y} \mid x \in\{1,2, \ldots, 10\} \wedge y \in \mathbb{Z} \wedge-4 \leq y \leq 1\right\}$. Then, for each combination of $C$ and $\gamma$, the LPO CV was applied to the RBF-SVM. For each subtest, the mean and standard deviation of accuracy was calculated. In case a range for $C$ and $\gamma$ with particularly high accuracy can be identified, the methodology can be repeated with a narrower grid. The RBFSVM parameters are selected according to the optimal accuracy. The resulting classifier was then used to classify unknown subviral particle tracks. As an example, the three tracks from the previous publication were classified as seemingly directed, moderate and chaotic. The algorithm is programmed in Python 3.7.

\section{Results}

Figure 2 shows the average accuracies in a color heat map as a function of the RBF-SVM parameters in logarithmic scaling. Blue represents a relatively low accuracy. In contrast, red stands for a relatively high accuracy. The minimum accuracy is about $53 \% \pm 18 \%$, whereas the maximum accuracy is about $88 \% \pm 13 \%$. A range for $2 \leq C \leq 20$ and $0.05 \leq \gamma \leq$ 0.4 is recognizable, which is characterized by particularly high accuracies and contains the maximum. 


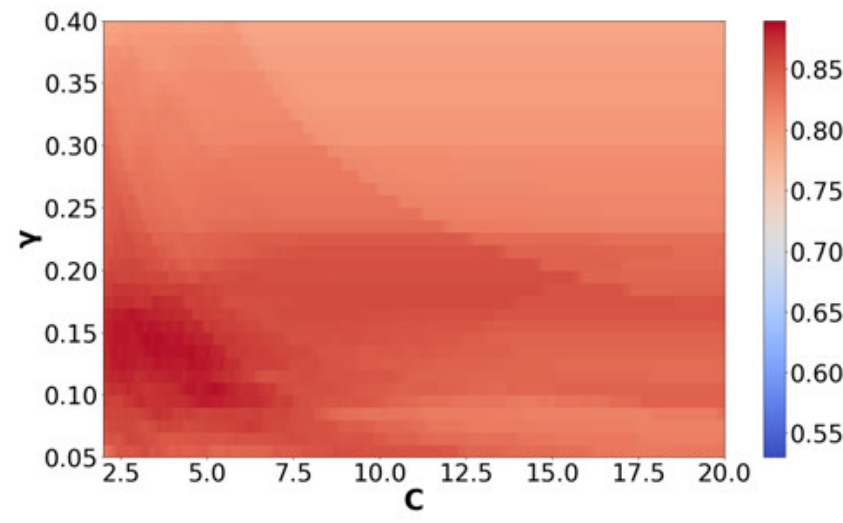

Figure 3: RBF-SVM parameter dependent average accuracies for $N=100$ are shown by means of a colour heat map as a function of the RBF-SVM parameters for a narrower grid with $2 \leq C \leq 20$ and $0.05 \leq \gamma \leq 0.4$.

In Figure 3, this area is examined in more detail with a narrower grid and linear scaling. Here the minimum accuracy is about $79 \% \pm 15 \%$. The maximum accuracy is about $89 \% \pm 13 \%$ for $C=3.2$ and $\gamma=0.14$. Further investigation of even narrower grid showed that in the range for $3.15 \leq$ $C \leq 3.24$ and $0.139 \leq \gamma \leq 0.144$ a plateau with the accuracy $89 \% \pm 13 \%$ occurs. Applying the methodology to an SVM with linear kernel achieves a unique maximum accuracy of $85 \% \pm 15 \%$ for $C=0.2$.

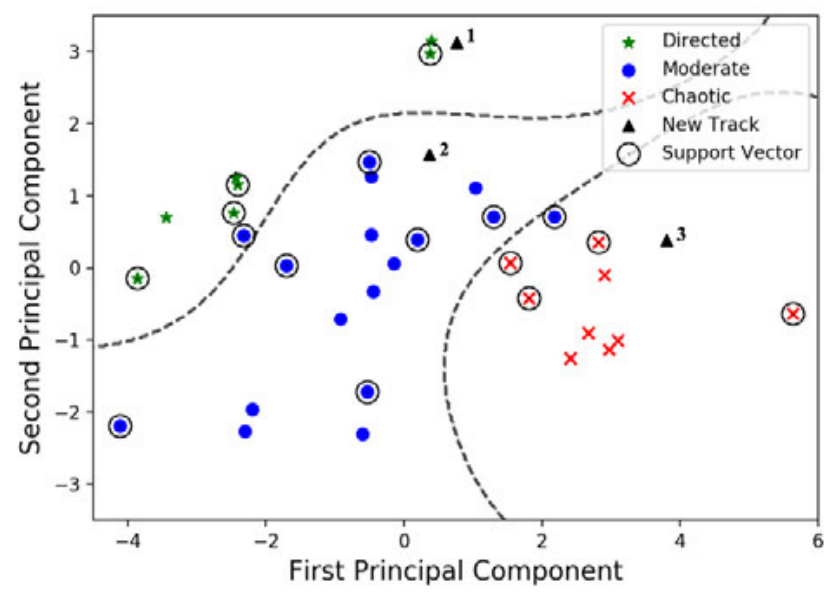

Figure 4: Scatter plot of the second principal component (PC) vs. the first $P C$ with the support vectors and the decision functions shown as dashed lines resulting from RBF-SVM with estimated parameters for $C=3.2$ and $\gamma=0.14$. New tracks with directed, moderate and chaotic motion patterns tested in modelled RBFSVM are marked with 1, 2 and 3.

Figure 4 shows the application of the estimated parameters for $C=3.2$ and $\gamma=0.14$ to the RBF-SVM by using methods and estimated parameters shown in a previous publication [5]. The results obtained with the algorithm for the dataset are presented as scatter plot that maps the second principal component (PC) versus the first PC (Fig. 4). The manually classified training data and the algorithmically reassigned track are illustrated in Figure 4. Additionally, resulting from the RBF-SVM, the support vectors are denoted by circles and the decision functions are shown as dashed lines.

\section{Discussion and conclusion}

Figure 2 shows that the presented method with broad grid search can obtain distinguishable results with a minimum accuracy of $53 \% \pm 18 \%$ and a maximum accuracy of $88 \% \pm 13 \%$. A range for $2 \leq C \leq 20$ and $0.05 \leq \gamma \leq 0.4$ can be identified in which the RBF-SVM parameters lie with the highest possible accuracy for classification of motion patterns of subviral particles. With these results, the methodology can be repeated with narrower grid (Fig. 3) provided that the accuracy-function is smooth, e.g. free of jumps, delta functions etc. This step is necessary because the LPO CV has a high iterative computational cost due to the over $4 * 10^{6}$ possible combinations of train and test dataset for a sample of size 33. With a narrower chosen grid, the iterations would increase by a factor of the combinations from $C$ and $\gamma$. To counteract this, the limitation of combinations to $N=100$ and stepwise approximation is used. The narrower grid, shown in Figure 1, covers high accuracies with a minimum value of $79 \% \pm 15 \%$. Here the maximum accuracy of $89 \% \pm 13 \%$ for $C=3.2$ and $\gamma=0.14$ is reached. The plateau in the range for $3.15 \leq C \leq 3.24$ and $0.139 \leq \gamma \leq 0.144$ indicates that the highest reasonable resolution of the grid search is applied. Overall, extremely good values for the accuracy of $89 \% \pm$ $13 \%$ can be obtained with the estimated RBF-SVM parameters for the classification of motion patterns of subviral particles using the method shown. The relatively high standard deviation is due to the small size of the test data sets of $p=7$. A wrong classification in test dataset for one iteration of the LPO already has a proportion of about $14 \%$. The choice of $p$, however, is capped due to increasing computational intensity and the need for a sufficiently large training data set. Minimizing $p$, in turn, results in a small variance in the determined accuracy for the available sample. This would have led to a less tightly constrainability of $C$ and $\gamma$. Therefore, the much simpler leave-one-out $\mathrm{CV}$ could not be used. The publication "Machine learning algorithm validation with a limited sample size" by Vabalas et al. [11] also shows that methods like k-fold CV are not sufficient to control overfitting for a small sample size. In contrast, nested CV, similar to the 
presented methodology, were unbiased regardless of sample size.

Figure 4 shows the application of the estimated parameters to the RBF-SVM for the classification of new tracks with unknown motion patterns. These are the same tracks used in a previous publication [5] for the classification of an SVM with linear kernel. While an accuracy of $85 \% \pm 15 \%$ could be achieved with linear kernel, the RBF-SVM achieves 4\%points better values of $89 \% \pm 13 \%$. Like the linear SVM, the RBF-SVM classifies all new tracks into the appropriate class of their motion patterns. The difference in accuracy is due to the higher complexity and better adaptability of the decision functions of the RBF-SVM.

The work shows that despite the small sample size, it is possible to identify a range for the optimal values of the RBF parameters $C$ and $\gamma$. Parameter estimation for the RBF-SVM using grid search with the LPO CV achieves high accuracies for classification of known motion patterns of subviral particles. This has also been confirmed when applied to new tracks with unknown motion patterns (Fig. 4). Even higher accuracies can be achieved with the RBF-SVM than with the linear SVM. However, the parameter estimation is accompanied by a high computational burden. This must be tolerated in order to achieve the most accurate results possible and to compensate for the small sample size. In future work, if the sample size should be increased, the applied method could be compared with less computationally intensive methods. Thus, it can be determined whether it needs an excessive expansion of the sample. Basically, complementary to our previous publication [5], this work shows that discrimination of the motion patterns of subviral particles is possible with the developed algorithm.

\section{Acknowledgments}

We want to thank the work group of Stephan Becker from the Institute for Virology, Marburg, Germany. Especially, we want to thank Olga Dolnik for providing fluorescence microscopic image sequences and her expertise in manually evaluating data for our research.

\section{Author Statement}

Research funding: The author state no funding involved. Conflict of interest: Authors state no conflict of interest. Informed consent: Informed consent has been obtained from all individuals included in this study. Ethical approval: The research related to human use complies with all the relevant national regulations, institutional policies and was performed in accordance with the tenets of the Helsinki Declaration, and has been approved by the authors' institutional review board or equivalent committee.

\section{References}

[1] Who, World Health Organization: "Ebola virus disease." World Health Organization: WHO, 23 Feb. 2021, www.who.int/news-room/fact-sheets/detail/ebola-virusdisease. Retrieved May 10, 2021.

[2] Schudt G, Klesnikova L, Dolnik O, Sodeik B, Becker S. LiveCell imaging of Marburg virus- infected cells uncovers actindependent transport of nucleocapsids over long distances. Proc. Nat. Acad. Sci. USA, 2013.

[3] Kienzle C, Schudt G, Becker S, Schanze T. Multiple subviral particle in fluorescence microscopy sequences. Bildverarbeitung für die Medizin. Springer-Verlag; 2014:330335.

[4] Rausch A, Müller D, Schanze T. Improvement of a subvira particle tracker by the use of a LAP-Kalman-algorithm. Current Directions in Biomedical Engineering; 2016:2.

[5] Schuhmann RM, Rausch A, Schanze T. Classification of movement patterns of subviral particles using a support vector machine, AUTOMED (in print); 2021

[6] M. J. Katz, E. B. George. Fractals and the Analysis of Growth Paths. Bulletin of Mathematical Biology; 1985:47:273-286.

[7] Rausch A, Kaak M, Müller D, Schanze T. Spline Interpolation Based Analysis of Subviral Particle Tracks in Fluorescence Image Sequences by Curvature Determination. Current Directions in Biomedical Engineering.

[8] Kaak M, Rausch A, Schanze T. Automatic Classification of the Movements of Directed and Undirected Subviral Particles. Current Directions in Biomedical Engineering; 2020:6.

[9] Cortes C, Vapnik V. Support-vector networks. Machine Learning; 1995:20(3):273-297.

[10] Celisse A, Robin S. Nonparametric density estimation by exact leave-p-out cross-validation. Computational Statistics \& Data Analysis; 2008:52:2350-2368.

[11] Vabalas A, Gowen E, Poliakoff E, Casson AJ, Machine learning algorithm validation with a limited sample size. PLOS ONE; 2019:14(11). 